\title{
Un congrès Nord-Sud à Oujda sur la recherche et l'enseignement de la physique
}
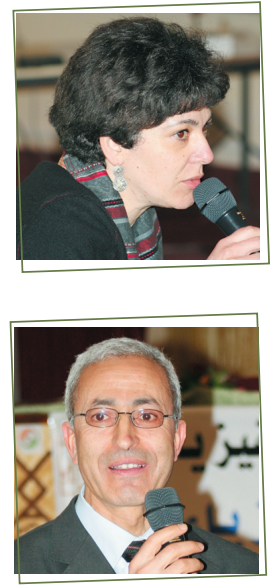

Les coprésidents du congrès.

Photo du haut Faïrouz Malek (CNRS, Grenoble, responsable de l'action Nord-Sud de la SFP).

Photo du bas : Jamal Derkaoui (Doyen de l'université d'Oujda, Maroc)

Les participants au congrès.

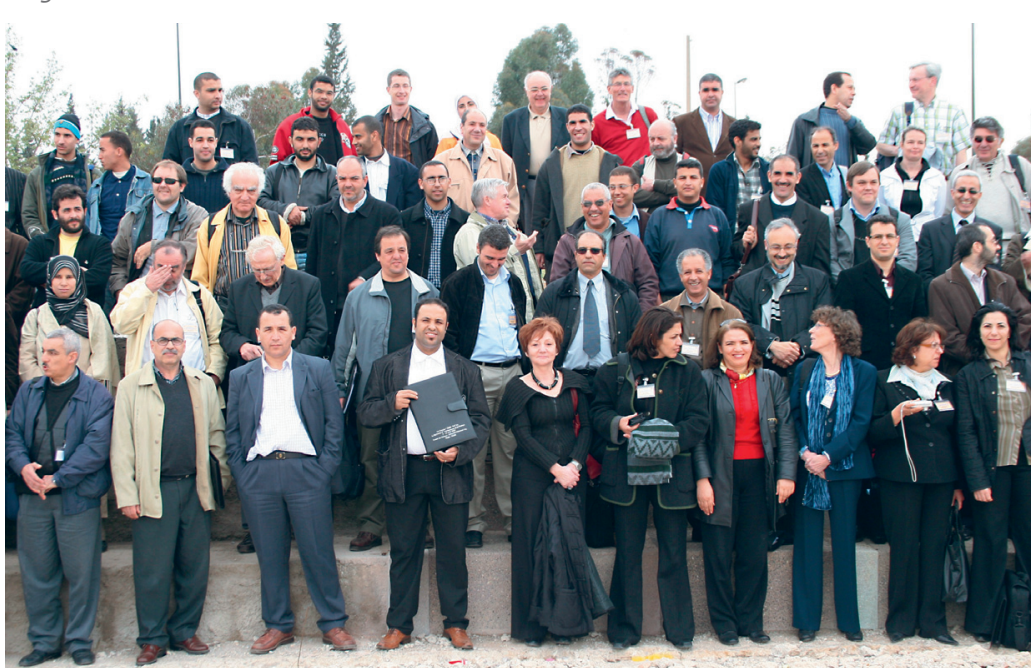

Un panorama non exhaustif de la recherche susceptible de donner lieu à des coopérations NordSud a été présenté, allant des grands équipements à la nanotechnologie. Quelques pistes pour la mise en place de réseaux de recherche en commun ont été identifiées et restent à préciser : citons les matériaux granulaires, les composants de la microélectronique ou les applications de l'optique. Les avantages d'un programme structurant autour d'un grand instrument pour le Maghreb ont été évoqués, ainsi que l'opportunité offerte par le synchrotron SESAME au Moyen-Orient.

Pour la formation des jeunes physiciens et des jeunes enseignants-chercheurs, un projet de création d'une école au Maghreb a été proposé et accueilli très favorablement. Cette école pourrait s'inspirer du modèle de l'école prédoctorale aux Houches qui, chaque année, dispense pendant deux semaines des cours au meilleur niveau de la recherche actuelle dans un domaine de physique assez large. Elle rassemblerait des scientifiques du Nord et du Sud (Maghreb, Afrique francophone et Afrique du Sud).

Le congrès s'est conclu par la mise sur pied d'un comité de suivi Nord-Sud chargé d'identifier des réseaux de coopération, de faire des propositions pour l'école au Maghreb et plus généralement de préparer un projet d'envergure d'actions communes dans le domaine de la physique entre la France et les pays d'Afrique francophone. Ce congrès a été l'occasion de rassembler les diverses associations de physique marocaines pour constituer une Société Marocaine de Physique. II a aussi permis de resserrer les liens entre la SFP et la Société Tunisienne de Physique, qui s'apprêtent à signer ensemble une convention de coopération.

Michèle Leduc, Faïrouz Malek et Roger Maynard

(1) Le programme, les présentations des conférenciers et les photographies du congrès, qui s'est tenu du 9 au 13 avril 2007 à I'Université Mohamed 1 er à Oujda, Maroc, peuvent être trouvés sur le site http://sfpnordsud.in2p3.fr/congres/ 